\title{
Incompressible representations of the Birman-Wenzl-Murakami algebra
}

\author{
Vincent Pasquier.
}

Service de Physique Théorique, C.E.A/ Saclay, 91191 Gif-sur-Yvette, France.

\begin{abstract}
We construct a representation of the Birman-Wenzl-Murakami algebra acting on a space of polynomials in $n$ variables vanishing when three points coincide. These polynomials are closely related to the Pfaffian state of the Quantum Hall Effect and to the components the transfer matrix eigenvector of a $\mathrm{O}(\mathrm{n})$ crossing loop model.
\end{abstract}

\section{Introduction and Conclusion}

This paper continues a preceding one [1] where we have studied the deformations of certain wave functions of the quantum Hall effect. There, the deformations were related to representations of the Temperley-Lieb algebra. Here, we consider yet another class whose deformation yields a representation of the Birman-Wenzl-Murakami (B.M.W.) algebra [2] 3].

Read and Rezayi [4] [5] have initiated the study quantum Hall wave functions related to parafermion currents in two-dimensional conformal field theory. They are symmetric (or antisymmetric) polynomials in $z_{1}, \ldots, z_{n}$ vanishing quadratically as any $k+1$ particles come to the same point. They model a quantum Hall state where the particles are gathered in clusters of $k$ and are expected to obey non-Abelian statistics.

Here, we are mainly considering the ground state which is the minimal degree nonzero symmetric polynomial satisfying the constraint. For a number of variables multiple of $k$, $n=k m$, it is obtained as a symmetrized sum of terms obeying the constraint and having the factorized expression:

$$
P\left(z_{1}, \ldots, z_{n}\right)=\prod_{i=1}^{n} \prod_{p=1}^{m-1} b_{i i+p}
$$


with $b_{i j}=z_{i}-z_{j}$, and the cyclic identification of the indices $\left(z_{i}=z_{i+n}\right)$ is assumed.

Feigin, Jimbo, Miwa and Mukhin [6] have independently considered these wave functions. Moreover, they have introduced a q-deformation obeying the cancellation condition 7]:

$$
P\left(z_{1}, z_{2}, \ldots, z_{k+1}\right)=0 \text { for } z_{i+1}=q^{2 i} z_{1}, 1 \leq i \leq k .
$$

In particular, the ground state is obtained by substituting $b_{i j} \rightarrow q z_{i}-q^{-1} z_{j}$ in (11).

This paper is a step towards the generalization of the condition (2) when the polynomials are no longer symmetrical. Namely, we consider polynomials in an infinite set of variables $z_{i}$, subject to the identification $z_{i+n}=q^{2 k} p^{2} z_{i}$, and obeying the cancellation condition:

$$
P\left(z_{i_{1}}=z, z_{i_{2}}=q^{2} z, \ldots, z_{i_{k+1}}=q^{2 k} z\right)=0 \text { for } i_{1}<i_{2} \ldots<i_{k+1} .
$$

The wave function (11) satisfies (3) , but is not the unique ground state. The claim is that the space of the ground states can be organized into a representation of the braid group. Here, we concentrate on the case $k=2$. We show that the polynomials of the minimal degree obeying (3) form an irreducible representation of the Birman-Wenzl-Murakami algebra.

¿From a different perspective, Di Francesco and Zinn-Justin [8] and Knutson and Zinn-Justin [9] have obtained the ground state eigenvector of a $O(1)$ loop model on the cylinder and derived from it combinatoric properties of certain algebraic varieties, in connection with conjectures of De Gier and Nienhuis [10]. Here, when $k=2$ and the cyclic condition $q^{2 k}=p^{-2}$ is satisfied, the degenerate ground states are the components of the Perron-Frobenius eigenvector of a statistical mechanics transfer matrix. Their sum is a symmetrical polynomial given by a deformation of the Pfaffian state [1]:

$$
\operatorname{Pf}\left(\frac{1}{q z_{i}-q^{-1} z_{j}}-\frac{1}{q z_{j}-q^{-1} z_{i}}\right) \prod_{i<j}^{n} \frac{\left(q z_{i}-q^{-1} z_{j}\right)\left(q z_{j}-q^{-1} z_{i}\right)}{z_{i}-z_{j}}
$$

The correspondence between the polynomials and the braid group relies on a representation of the permutations inherited from a solution of the Yang-Baxter equation. The starting point is a wave function which is transformed in the same way by permuting the coordinates or by acting with the Yang-Baxter matrix. The approach is similar, but technically more involved than [1] because the polynomials obeying (31) decompose into three pieces instead of two:

$$
P=S_{0}+\left(q z_{i}-q^{-1} z_{i+1}\right)\left(S_{1}+\left(p z_{i}-p^{-1} z_{i+1}\right) S_{2}\right) .
$$


The three polynomials of the decomposition satisfy the constraint, and the polynomials $S_{i}$ are symmetrical under the exchange of $z_{i}$ and $z_{i+1}$. The generators of the B.M.W. algebra can be identified with the projectors onto the three pieces of (15).

The paper is divided into two sections. In the first one we recall the definition of the B.M.W. algebra, we define the representation which we need and we exhibit a basis in terms of tangles. In the second one, we consider the polynomial space introduced above and we identify a basis of these polynomials with the tangle basis. The appendix B studies the Brauer algebra which is the rational limit of the B.M.W. algebra. We show in the appendix $\mathrm{C}$ that the Temperley-Lieb structure is intimately related to $k=2$.

We hope to return to the $k>2$ cases and to the higher degree polynomials (excited states) elsewhere.

\subsection{Acknowledgements}

I have benefited from discussions with Philippe Di Francesco, Vincent Lafforgue and N. Read.

\section{Birman-Wenzl-Murakami algebra}

The B.M.W. algebra $\mathcal{B}_{n}$ is generated by braid generators $t_{i}$ and Temperley Lieb generators $e_{i}$, for $1 \leq i \leq n-1$, obeying the relations:

$$
\begin{aligned}
& t_{i} t_{i+1} t_{i}=t_{i+1} t_{i} t_{i+1}, \quad t_{i} t_{j}=t_{j} t_{i}, \text { if }|i-j| \geq 2, \\
& e_{i} e_{i \pm 1} e_{i}=e_{i}, \quad e_{i} e_{j}=e_{j} e_{i}, \text { if }|i-j| \geq 2, \\
& t_{i}-t_{i}^{-1}=\epsilon\left(1-e_{i}\right), \\
& e_{i} t_{i}=t_{i} e_{i}=a e_{i}, \\
& e_{i}^{2}=\tau e_{i}, \\
& e_{i} t_{i \pm 1} e_{i}=a^{-1} e_{i}, \\
& t_{i \pm 1} t_{i} e_{i \pm 1}=e_{i} t_{i \pm 1} t_{i}=e_{i} e_{i \pm 1},
\end{aligned}
$$

with $a=p^{-1} q^{-2}$ and:

$$
\begin{aligned}
a & =p^{-1} q^{-2}, \epsilon=p-p^{-1} \\
\tau & =1-\frac{a-a^{-1}}{\epsilon}=\frac{p q-p^{-1} q^{-1}}{p-p^{-1}}\left(q+q^{-1}\right) .
\end{aligned}
$$

Notice that there is an algebra isomorphism from $\mathcal{B}_{n}(\tau, a, \epsilon)$ to $\mathcal{B}_{n}\left(\tau, a^{-1},-\epsilon\right)$ defined by $e_{i} \rightarrow e_{i}, t_{i} \rightarrow t_{i}^{-1}$. 
This algebra can be endowed with a trace defined by:

$$
\begin{aligned}
& \operatorname{tr}(1)=1, \\
& \operatorname{tr}\left(x t_{n}\right)=\frac{1}{\tau a} \operatorname{tr}(x) \text { for } x \in \mathcal{B}_{n-1}, \\
& \operatorname{tr}\left(x e_{n}\right)=\frac{1}{\tau} \operatorname{tr}(x) \text { for } x \in \mathcal{B}_{n-1} .
\end{aligned}
$$

The B.M.W. algebra $\mathcal{B}_{n}$ has a natural representation in terms of tangles, modulo Kaufman skein relations [12 13. Consider a rectangle with $2 n$ points at its boundary. The $n$ points denoted $1,2, \ldots, n$ are disposed from left to right on the lower side and the $n$ points denoted $\overline{1}, \overline{2}, \ldots, \bar{n}$ are disposed above $1,2, \ldots, n$ on the upper side. A tangle is made of $n$ strands projected onto the rectangle connecting pairwise the $2 n$ points at the boundary. We denote the strand connecting $k$ to $l$ by $\left(\begin{array}{l}k \\ l\end{array}\right)$.

The tangles $\pi_{1}, \pi_{2}$ are composed by placing the rectangle $\pi_{2}$ on top of $\pi_{1}$ so as to obtain a rectangle where the lower points $k$ of $\pi_{2}$ coincide with the upper points $\bar{k}$ of $\pi_{1}$. The tangle denoted $\pi_{1} * \pi_{2}$ is obtained by joining into a single stand the strand of $\pi_{1}$ ending at $\bar{k}$ with the strand of $\pi_{2}$ ending at $k$.

The identity tangle is made of $n$ vertical strands connecting $\bar{k}$ to $k$ :

$$
I d=\left(\begin{array}{cccc}
\overline{1} & \overline{2} & \ldots & \bar{n} \\
1 & 2 & \ldots & n
\end{array}\right)
$$

The generator $t_{k}$ is obtained from the identity by exchanging the two ends $k$ and $k+1$ in such a way that the strand $\left(\begin{array}{c}\bar{k} \\ k+1\end{array}\right)$ crosses over $\left(\begin{array}{c}\overline{k+1} \\ k\end{array}\right)$. Similarly, the generators $e_{k}$ is made of $n-2$ strands connecting $l$ to $\bar{l}$ for $l \neq k, k+1$, two disjoint strands, one above connecting $\bar{k}$ to $\overline{k+1}$, and one below connecting $k$ to $k+1$.

The defining relations of the algebra (6) can be recast into skein relations allowing to identify tangles. A tangle is multiplied by a factor $a^{ \pm 1}$ each time a loop is untwisted and a close loop can be removed by multiplying the tangle by a factor $\tau$. The relation $t_{i}-t_{i}^{-1}=\epsilon\left(1-e_{i}\right)$ enables to relate over-crossings to under-crossings. The trace closes the rectangle into a cylinder by identifying the upper with the lower edge.

\subsection{Representation of the B.W.M. algebra on words}

We consider the case $n$ even. A Hilbert space $\mathcal{H}_{n}$ is defined by acting with $\mathcal{B}_{n}$ to the left on the minimal projector $\alpha=e_{1} e_{3} \ldots e_{n-1}$.

A basis is given by the reduced words:

$$
\pi=\left(t_{c_{n-1}}^{-1} t_{c_{n-1}+1}^{-1} \ldots e_{n-1}\right) \ldots\left(t_{c_{2 p+1}}^{-1} t_{c_{2 p+1}+1}^{-1} \ldots e_{2 p+1}\right) \ldots e_{1},
$$


with $0 \leq p \leq \frac{n}{2}-1$ and $1 \leq c_{2 p+1} \leq 2 p+1$, and each sequence reduces to $e_{2 p+1}$ when $c_{2 p+1}=2 p+1$. There is an order relation: $\pi>\pi^{\prime}$ if $\pi$ is written $b \pi^{\prime}$ with $b$ a monomial in $t_{i}^{-1}$.

Basis states can be identified with their tangle. The upper part of the tangle is made of $\frac{n}{2}$ arches connecting $\overline{2 k-1}$ to $\overline{2 k}$ and can be ignored. The lower $n$ points $1,2, \ldots, n$ are connected pairwise in all possible ways. When two strands, $\left(\begin{array}{l}a_{1} \\ b_{1}\end{array}\right)$ and $\left(\begin{array}{l}a_{2} \\ b_{2}\end{array}\right)$ with $a_{1}<b_{1}$, $a_{2}<b_{2}$ and $b_{1}<b_{2}$ cross, $\left(\begin{array}{l}a_{2} \\ b_{2}\end{array}\right)$ crosses over $\left(\begin{array}{l}a_{1} \\ b_{1}\end{array}\right)$. A basis state is therefore characterized by the sequence of strands:

$$
\pi=\left(\begin{array}{cccc}
a_{1} & a_{2} & \ldots & a_{n} \\
b_{1} & b_{2} & \ldots & b_{n}
\end{array}\right)
$$

with $a_{1}<a_{2}<\ldots<a_{n}, a_{i}<b_{i}$.

The basis states (91) can be recursively identified with tangles as follow: The tangle representing the word $\pi=\left(t_{c_{n-1}}^{-1} \ldots e_{n-1}\right) \pi^{\prime}$ with $\pi^{\prime} \in \mathcal{H}_{n-2}$ is obtained by pushing by one unit to the right the strand ends of $\pi^{\prime}$ larger or equal to $c_{n-1}$, and by inserting a strand $\left(\begin{array}{c}c_{n-1} \\ n\end{array}\right)$ upon the tangle obtained in this way.

Consider the maximally crossed state $\rho$ characterized by the condition $t_{i}^{-1} \rho=t_{\frac{n}{2}+i}^{-1} \rho$ for $1 \leq i \leq \frac{n}{2}-1$ :

$$
\rho=\left(\begin{array}{cccc}
1 & 2 & \ldots & \frac{n}{2} \\
\frac{n}{2}+1 & \frac{n}{2}+2 & \ldots & n
\end{array}\right)
$$

Another way to obtain the basis states is to act on $\rho$ with the generators $t_{i}^{ \pm 1}$, imposing that the number of crossings decreases by one unit each time one acts with $t_{i}^{ \pm 1}$. The possible actions are given by:

$$
\begin{aligned}
t_{i}^{-1}\left(\begin{array}{cc}
i & i+1 \\
j & k
\end{array}\right) & =\left(\begin{array}{cc}
i & i+1 \\
k & j
\end{array}\right) \text { if } k>j, \\
t_{i}\left(\begin{array}{ccc}
j & \ldots & i \\
i+1 & \ldots & k
\end{array}\right) & =\left(\begin{array}{ccc}
j & \ldots & i+1 \\
i & \ldots & k
\end{array}\right) \\
t_{i}^{-1}\left(\begin{array}{ccc}
j & \ldots & k \\
i & \ldots & i+1
\end{array}\right) & =\left(\begin{array}{ccc}
j & \ldots & k \\
i+1 & \ldots & i
\end{array}\right) .
\end{aligned}
$$

A different order relation than the preceding one is now $\pi>\pi^{\prime}$ if $\pi$ is written $b \pi^{\prime}$ with $b$ a monomial in $t_{i}^{ \pm 1}$. Thus one must be able to go from $\pi^{\prime}$ to $\pi$ by a sequence of untwists. The only minimal tangle is $\rho$ and all the basis states can be reached from it by this procedure. We call this order the crossing order to differentiate it from the first one. 
By identifying the two vertical sides of the rectangle, we can view the labels $1,2, \ldots, n$ as arranged cyclically around a circle. We define $\sigma$, the operator which acts by cyclically permuting the indices of a tangle $i \rightarrow i-1$ :

$$
\sigma=a t_{n}^{-1} t_{n-1}^{-1} \ldots t_{1}^{-1} .
$$

The normalization factor can be determined by a straightforward check that $\sigma^{-2} \alpha=\alpha$.

Note that the application of $\sigma$ on a reduced word $\pi$ spoils the crossing rules and the relations (6) need to be used to put the word in its canonical form back. Nevertheless, $\sigma$ is a triangular matrix for the crossing order: $\sigma \pi=\pi_{\sigma}+$ tangles $>\pi$, where $\pi_{\sigma}$ is the basis state obtained from $\pi$ by the substitution $i \rightarrow i-1$. It follows from this that the line $\rho$ of the matrix representing $\sigma$ has only zeros except a 1 for the diagonal element.

A Hermitian conjugation is defined by $t_{i}^{*}=t_{i}^{-1}, e_{i}^{*}=e_{i}, p^{*}=p^{-1}, q^{*}=q^{-1}$, and a scalar product as:

$$
\pi^{*} \pi^{\prime}=\left\langle\pi \mid \pi^{\prime}\right\rangle \alpha .
$$

\subsubsection{Structure of the matrices $e_{i}, t_{i}$ and dual basis}

In the basis (9), the matrices $e_{i}, t_{i}$ have the following structure. The basis states can be split into the tangles $\Pi_{0}^{i}$ with a strand $\left(\begin{array}{c}i \\ i+1\end{array}\right)$ connecting $i$ to $i+1$, the tangles $\Pi_{1}^{i}$ where the two strands ending at $i$ and $i+1$ cross and the tangles $\Pi_{2}^{i}$ where they do not cross: $t_{i} \Pi_{2}^{i}=\Pi_{1}^{i}$. We identify the tangles with the projector onto these tangles, $\Pi_{0}^{i} \oplus \Pi_{1}^{i} \oplus \Pi_{2}^{i}=1$. Thus, $\left(\tau-e_{i}\right) \Pi_{0}^{i}=\left(t_{i}-a\right) \Pi_{0}^{i}=0$ and $\left(\Pi_{1}^{i}+\Pi_{2}^{i}\right) e_{i}=0$. Using the relation $e_{i}\left(t_{i}-a\right)=0$, the matrices $t_{i}, e_{i}$ are given the following block matrix expression:

$$
e_{i}=\left(\begin{array}{ccc}
\tau & a v & v \\
0 & 0 & 0 \\
0 & 0 & 0
\end{array}\right), \quad t_{i}=\left(\begin{array}{ccc}
a & -a \epsilon v & 0 \\
0 & \epsilon & 1 \\
0 & 1 & 0
\end{array}\right)
$$

with,

$$
v=\Pi_{0}^{i} e_{i} \Pi_{2}^{i} .
$$

Let us now consider the dual basis of (9). Its basis elements $\bar{\pi}$ are labeled by reduced words $\pi$ and are defined by the relation:

$$
\bar{\pi}\left(\pi^{\prime}\right)=\delta_{\pi, \pi^{\prime}} .
$$


If $\psi$ is an element of the B.M.W algebra, we have the duality relation:

$$
\bar{\pi} \psi\left(\pi^{\prime}\right)=\bar{\pi}\left(\psi \pi^{\prime}\right)
$$

so that we can generate the whole dual basis upon acting with the B.M.W. algebra on the generator $\bar{\alpha}$. The matrices $t_{i}$ (15) are such that the column indexed by the tangle $\pi \in \Pi_{2}^{i}$ has only zeros except for a 1 at the intersection with the line indexed by $t_{i} \pi \in \Pi_{1}^{i}$. Similarly, the line $t_{i} \pi \in \Pi_{1}^{i}$ has only zeros except for a 1 at the intersection with the column $\pi \in \Pi_{2}^{i}$. ¿From this, using the fact that the the basis elements are obtained by successive action of the generators $t_{i}$ on $\Pi_{2}^{i}$, we deduce that $\bar{\pi}$ can be identified with the reduced expression of $\pi$ written in the reverse order:

$$
\bar{\pi}=\bar{\alpha} \ldots\left(t_{2 p}^{-1} \ldots t_{a_{2 p+1}+1}^{-1} t_{a_{2 p+1}}^{-1}\right) \ldots\left(t_{n-2}^{-1} \ldots t_{a_{n}}^{-1}\right) .
$$

A similar argument shows that the same holds for words constructed using $\bar{\rho}$ instead of $\bar{\alpha}$ as a generator.

\section{Polynomial representations}

\subsection{Projectors from the Yang-Baxter equation}

Let us consider the Yang-Baxter equation:

$$
Y_{12}(z) Y_{23}(z w) Y_{12}(w)=Y_{23}(w) Y_{12}(z w) Y_{23}(z) \text {. }
$$

It is possible to define an operator $Y_{i i+1}(z)$ solution of the Yang-Baxter equation in terms of the algebra (6) [14] 15]:

$$
D(z) Y_{i i+1}(z)=(z-1) p^{-1} a^{-1} t_{i}-\epsilon\left(1-p^{-1} a^{-1}\right)+\left(z^{-1}-1\right) t_{i}^{-1},
$$

where the normalization factor $D(z)$ is chosen so that $Y\left(z^{-1}\right) Y(z)=1$. A possible choice

for $D(z)$ is to require that $Y_{i i+1} e_{i}=1$. Then, $D(z)$ is factorized as $D(z)=\left(z-q^{2}\right)(z-$ $\left.p^{2}\right) p^{-1} z^{-1}$.

This allows us to identify consistently the Yang-Baxter matrix $Y_{i i+1}$ with the permutation of the variables $z_{i}, z_{i+1}$ of a vector $\Psi\left(z_{1}, \ldots, z_{n}\right)$ parameterized by $z_{i}$ :

$$
Y_{i i+1}\left(\frac{z_{i+1}}{z_{i}}\right) \Psi=\Psi k_{i i+1}
$$


where $k_{i i+1}$ acts by permuting the variables $z_{i}$ and $z_{i+1}$ of the expressions located left of it.

We want to use the relation (22) to constrain operators $\bar{t}_{i}$ and $\bar{e}_{i}$ which act on the components $\bar{\pi}$ of $\Psi$ and are dual to the $t_{i}, e_{i}$ :

$$
t_{i} \Psi=\Psi \bar{t}_{i}, \quad e_{i} \Psi=\Psi \bar{e}_{i} .
$$

(22) does not allow to obtain a closed expression for $\bar{t}_{i}$ and $\bar{e}_{i}$, but gives only a partial information about them. By multiplying (22) by $e_{i}$ to the left, we obtain: $e_{i} \Psi\left(k_{i i+1}-\right.$ $1)=0$, which, with (23), implies that $\bar{e}_{i}$ projects onto polynomials symmetric under the exchange of $z_{i}, z_{i+1}$.

If we multiply (22) by $e_{i}-\tau$ to the left, we obtain:

$$
\left(e_{i}-\tau\right) \Psi k_{i i+1}=\frac{q z_{i+1}-q^{-1} z_{i}}{q z_{i}-q^{-1} z_{i+1}} \frac{\left(z_{i+1}-z_{i}\right) t_{i}+\left(p-p^{-1}\right) z_{i}}{p z_{i}-p^{-1} z_{i+1}}\left(e_{i}-\tau\right) \Psi .
$$

Since the left hand side is a polynomial, this implies that $e_{i}-\tau$ projects $\Psi$ onto its components divisible by $q z_{i}-q^{-1} z_{i+1}$. It is useful to introduce the notation $b_{i j}=q z_{i}-$ $q^{-1} z_{j}$. Thus,

$$
\begin{aligned}
\bar{\psi} \bar{e}_{i}=\tau \bar{\psi} & \Leftrightarrow \bar{\psi} \text { is symmetrical under the exchange of } z_{i}, z_{i+1} \\
\bar{\psi} \bar{e}_{i}=0 & \Leftrightarrow \bar{\psi} \text { is divisible by } b_{i i+1} .
\end{aligned}
$$

$\bar{e}_{i}$ projects onto the symmetrical polynomials under the exchange of $z_{i}$ and $z_{i+1}$, orthogonally to the polynomials divisible by $b_{i i+1}$. If we restrict to the components $\bar{\psi} \bar{e}_{i}=0$, the action of the projectors $\bar{t}_{i}-p$ and $\bar{t}_{i}+p^{-1}$ acquire the expressions:

$$
\begin{aligned}
\bar{t}_{i}-p & =-b_{i i+1}^{-1} \frac{p z_{i+1}-p^{-1} z_{i}}{z_{i+1}-z_{i}}\left(1+k_{i i+1}\right) b_{i i+1}, \\
\bar{t}_{i}+p^{-1} & =b_{i i+1}^{-1}\left(1-k_{i i+1}\right) \frac{p z_{i}-p^{-1} z_{i+1}}{z_{i}-z_{i+1}} b_{i i+1},
\end{aligned}
$$

Another way to view the action of the generators $t_{i}$ on polynomials is to use the affine-Hecke relations:

$$
\begin{aligned}
z_{i} \bar{t}_{j} & =\bar{t}_{j} z_{i} \text { if } i \neq j, j+1 \\
z_{i+1} \bar{t}_{i} & =\bar{t}_{i}^{-1} z_{i}
\end{aligned}
$$

to commute $\bar{t}_{i}^{ \pm 1}$ through the polynomial and to make use of the relation $b_{i i+1} \bar{t}_{i}=-p^{-1} b_{i i+1}$ when $b_{i i+1}$ is the only monomial left. 


\subsection{Statement of the problem}

Let us consider a vector $\Psi$ :

$$
\Psi=\sum_{\pi} \pi \bar{\pi}\left(z_{i}\right)
$$

constructed in the following way. The vectors $\pi$ are the basis vector of $\mathcal{H}_{n}$ indexed by $n$ even on which the B.M.W. algebra acts to the left. $\bar{\pi}$ are certain basis elements of a space $\mathcal{M}_{n}$ of homogeneous polynomials of degree $n\left(\frac{n}{2}-1\right)$ in the variables $z_{1}, z_{2}, \ldots, z_{n}$. We want to determine an action of the affine B.M.W. algebra on the polynomials, generated by the operators $\bar{t}_{i}$ and $\bar{\sigma}$ acting to the right and determine the coefficients $\bar{\pi}$ in such a way that both actions give the same result on the vector $\Psi$ :

$$
\begin{aligned}
& \Psi \bar{t}_{i}=t_{i} \Psi \\
& \Psi \bar{\sigma}=\sigma \Psi
\end{aligned}
$$

Said differently, we look for a dual action of the affine B.M.W. algebra acting on polynomials. Unless we specify it, we address this problem for a generic value of the deformation parameters, for which the B.M.W. algebra is semisimple.

We verify that this problem has a solution in the case $n=4$ in the appendix A.

\subsection{The property defining the polynomial space}

We consider a space of polynomials in an infinite number of variables $z_{i}$, where $z_{i+n}$ is identified with $a^{-2} z_{i}$, for $a=p^{-1} q^{-2}$. These polynomials are constructed as linear combinations of the monomials $z^{\mu}=z_{1}^{\mu_{1}} \ldots z_{n}^{\mu_{n}}$ with a total degree $|\mu|$ fixed. The cyclic operator $\bar{\sigma}$ acts as:

$$
\bar{\psi} \bar{\sigma}\left(z_{i}\right)=a^{\frac{2|\mu|}{n}} \bar{\psi}\left(z_{i+1}\right)
$$

where the normalization is such that $\bar{\sigma}^{n}=1$.

We require that these polynomials obey the property $(\mathrm{P})$ :

$$
(\mathrm{P}): \quad \bar{\psi}\left(z_{i}=z, z_{j}=q^{2} z, z_{k}=q^{4} z\right)=0, \text { if } i, j, k, \text { are cyclically ordered. }
$$

In order for $(\mathrm{P})$ to be compatible with the cyclic identification, $\bar{\psi}$ must vanish for the triplets: $\left(z_{i}, z_{j}, z_{k}\right)=\left(z, q^{2} z, q^{4} z\right),\left(z, p^{2} z, q^{2} p^{2} z\right)$ and $\left(z, q^{2} z, q^{2} p^{2} z\right)$ when $1 \leq i<j<$ $k \leq n$. 
$\mathcal{M}_{n}$ is the space of minimal degree polynomials satisfying $(\mathrm{P})$. We shall show that a polynomial belonging to $\mathcal{M}_{n}$ can be split in a unique way as:

$$
\bar{\psi}=S_{0}+b_{i i+1}\left(S_{1}+\left(p z_{i}-p^{-1} z_{i+1}\right) S_{2}\right),
$$

where the three polynomials in the decomposition belong to $\mathcal{M}_{n}$, and $S_{0}, S_{1}, S_{2}$ are symmetrical under the exchange of $z_{i}$ and $z_{i+1}$. To understand the origin of the second projector, assume that $\bar{\psi}$ is divisible by $b_{i i+1}: \bar{\psi}=b_{i i+1} \bar{\phi}$. It is then possible to act on $\bar{\psi}$ with the projectors (26) $)$, preserving the property $(\mathrm{P})$. The factor $\left(q z_{i}-q^{-1} z_{i+1}\right)\left(p z_{i}-p^{-1} z_{i+1}\right)$ in the definition of $\bar{\psi}\left(\bar{t}_{i}+p^{-1}\right)$ ensures that this polynomial vanishes when $z_{i+1}=q z_{i}$, or $z_{i+1}=p z_{i}$, and therefore obeys $(\mathrm{P})$.

The two projectors (26) enable to achieve the decomposition of the factor proportional to $b_{i i+1}$ in (32). Thus, at this point we can write:

$$
\begin{aligned}
\mathcal{M}_{n} & =\mathcal{M}_{n}^{0} \oplus \mathcal{M}_{n}^{1} \oplus \mathcal{M}_{n}^{2} \\
& =S_{0}+b_{12}\left(S_{1}+\left(p z_{1}-p^{-1} z_{2}\right) S_{2}\right)
\end{aligned}
$$

with $S_{1}, S_{2}$ symmetrical under the exchange of $z_{1}, z_{2}$, and $\mathcal{M}_{n}^{0}$ is a complementary space to the polynomials in $\mathcal{M}_{n}$ divisible by $b_{12}=q z_{1}-q^{-1} z_{2}$. Since $S_{0} \in \mathcal{M}_{n}^{0}$ is defined up to a polynomial divisible by $b_{12}$, it remains to prove that it can be chosen symmetrical under the exchange of $z_{1}, z_{2}$. The section 3.4 is devoted to prove this fact.

By applying (P) to $z_{1}, z_{2}, z_{i}$, we define a projection from $\mathcal{M}_{n} \rightarrow \mathcal{M}_{n-2}$ as:

$$
E^{\prime}(\bar{\psi})=c^{\prime} \frac{1}{\phi\left(z, z_{i}\right)} \bar{\psi}\left(z_{1}=z, z_{2}=q^{2} z, z_{i}\right)
$$

where $\phi\left(z, z_{i}\right)=\prod_{i=3}^{n}\left(q^{4} z-z_{i}\right)\left(p^{2} q^{2} z-z_{i}\right)$, and $c^{\prime}$ is a normalization constant. $E^{\prime}\left(\mathcal{M}_{n}\right) \subset$ $\mathcal{M}_{n-2}$, and using a recursion argument on $n$, the degree of the polynomials in $\mathcal{M}_{n}$ is $n\left(\frac{n}{2}-1\right)$.

Let us construct a basis of polynomials in correspondence with the dual states $\bar{\pi}$ (17). A state obeying $(\mathrm{P})$ with the correct degree is the maximally crossed state given by:

$$
\bar{\rho}=\prod_{i} \prod_{k=1}^{\frac{n}{2}-1} b_{i i+k}
$$

with the cyclic identifications understood and $b_{i j}=q z_{i}-q^{-1} z_{j}$.

One has $\bar{\rho} t_{i}=\bar{\rho} t_{\frac{n}{2}+i}$, it is therefore consistent to construct a basis of polynomials $\bar{\pi}$, by acting with the operators $\bar{t}_{i}, \bar{t}_{i}^{-1}$ on $\bar{\rho}$ as in (12). 
Since the generators $\bar{t}_{i}, \bar{t}_{i}^{-1}$ (26) act on polynomials divisible by $b_{i i+1}$, we must verify that the polynomial upon which one acts with $\bar{t}_{i}^{ \pm 1}$ is always divisible by $b_{i i+1}$. We show here a more general result.

If a tangle $\pi$ has consecutive points $j, j+1, \ldots, k$ with no strands connecting any two among them, we say that $j$ and $k$ are matched, if not they are split. In particular, two consecutive points are either matched, or connected by a strand. Let us show that when $j$ and $k$ are matched, the polynomial $\bar{\pi}$ is divisible by $b_{j k}$.

The property is true for $\bar{\rho}$, and we can use a recursion argument on the crossings. If two points are split in $\pi<\pi^{\prime}$, they are split in $\pi^{\prime}$, otherwise the number of crossings would not decrease continuously when going from $\pi$ to $\pi^{\prime}$. The only way $t_{i}$ splits $j<k$ from $k$ is when $i=j-1$, or $i=k$. Using the fact that $t_{i}$ commutes with $b_{i k} b_{i+1 k}\left(b_{k i} b_{k i+1}\right)$ as well as $b_{k l}$ for $k$ and $l \neq i, i+1$, we deduce that when $i$ is matched with $k$ the polynomial $\bar{\pi}$ is divisible by $b_{i k}$.

Once we have identified a basis of polynomial with the dual basis of $\mathcal{H}_{n}$, we have determined an action of $\mathcal{B}_{n}$ on $\mathcal{M}_{n}$. We still need to verify that this action is consistent with the decomposition (32), and in particular that $e_{i} \Psi$ is symmetrical under the exchange of $z_{i}$ and $z_{i+1}$.

The basis elements can be ordered by their highest monomial $z^{\lambda}$ (see [1]), and this ordering is compatible with the first ordering defined on the tangles. The highest monomial of the basis is given by $\lambda=(n-2, n-2, n-4, n-4, \ldots, 0,0)$.

\subsection{The projectors $\bar{e}_{i}$ and the full duality relation}

We obtain an expression of $\bar{e}_{1}$ as an operator acting on the components $\Pi_{0}^{1}$ having the two indices 1,2 connected by a link (split). The argument is essentially an adaptation to this case of the proof of Di Francesco and Zinn-Justin [8].

¿From the definition (25) of $\bar{e}_{1}$, we know that it is null on $\Pi_{1,2}^{1}$. Consider $\bar{\pi}_{0} \in \Pi_{0}^{1}$. The action of $\bar{e}_{1}$ on $\bar{\pi}_{0}$ is fully determined by the condition that $\bar{\pi}_{0} \bar{e}_{1}=\tau \bar{\pi}_{0}+\bar{\pi}_{1}$ is symmetrical under the exchange of $z_{1}, z_{2}$, and $\bar{\pi}_{1}$ is divisible by $b_{12}$.

We define the projector:

$$
\bar{f}_{1}=\left(1-k_{12}\right) \frac{1}{z_{1}-z_{2}}\left(p z_{1}-p^{-1} z_{2}\right)\left(q z_{1}-q^{-1} z_{2}\right) \frac{q+q^{-1}}{p-p^{-1}} .
$$

$\bar{f}_{1}$ raises the degree of the polynomial upon which it acts by one, its kernel are the symmetric polynomials in $z_{1}, z_{2}$, and $\bar{f}_{1}^{2}=\left(z_{1}+z_{2}\right) \tau \bar{f}_{1}$. The complementary projector: $\left(z_{1}+z_{2}\right) \tau-\bar{f}_{1}$ projects onto a polynomial symmetrical under the exchange of $z_{1}, z_{2}$.

Consider the following tangle $\pi_{0} \in \Pi_{0}^{1}$ : 


$$
\begin{aligned}
\pi_{0}=\left(\begin{array}{cccc}
1 & 3 & \ldots & \frac{n}{2}+1 \\
2 & \frac{n}{2}+2 & \ldots & n
\end{array}\right) & =t_{2} t_{3} \ldots t_{\frac{n}{2}} \rho \\
=a e_{1} t_{2}^{-1} \ldots t_{i-1}^{-1} t_{i+1} \ldots t_{\frac{n}{2}} \rho & =e_{1} t_{1}^{-1} t_{2}^{-1} \ldots t_{i-1}^{-1} t_{i+1} \ldots t_{\frac{n}{2}} \rho,
\end{aligned}
$$

for $2 \leq i \leq \frac{n}{2}$. With the diagonal element $e_{\pi_{0} \pi_{0}}=\tau$, the second line of (37) exhausts all the matrix elements $e_{\pi_{0} \pi}$ of the line $\pi_{0}$ of $e_{1}$. By duality, we must have:

$$
\bar{\pi}_{0} \bar{e}_{1}=\sum_{\pi} e_{\pi_{0} \pi} \bar{\pi} .
$$

If (25) holds, the right hand side of this equality must be a symmetrical polynomial in $z_{1}, z_{2}$. If we set:

$$
\bar{\pi}_{2}=\sum_{i=2}^{\frac{n}{2}} \bar{\rho} \bar{t}_{\frac{n}{2}} \ldots \bar{t}_{i+1} \bar{t}_{i-1}^{-1} \ldots \bar{t}_{2}^{-1},
$$

this amounts to show that:

$$
\tau \bar{\pi}_{0}+\bar{\pi}_{2}\left(a+\bar{t}_{1}^{-1}\right)
$$

is a symmetrical polynomial in $z_{1}, z_{2}$.

Let us consider the polynomial:

$$
\bar{\pi}^{\prime}=\bar{\pi}_{0} \bar{f}_{1}+\bar{\pi}_{2}\left(a+\bar{t}_{1}^{-1}\right)\left(z_{1}+z_{2}\right)+q^{-1} \bar{\pi}_{2}\left(p-\bar{t}_{1}\right) b_{21} .
$$

The third term of the above sum is symmetrical from the definition (26) of $p-\bar{t}_{1}$. From the fact that $\bar{f}_{1}-\left(z_{1}+z_{2}\right) \tau$ projects on a symmetric polynomial, the sum of the two first terms, and thus the hole expression, is symmetrical if and only if (40) holds. After substituting the expressions (26) of $\bar{t}_{1}$ and (36) of $\bar{f}_{1}$, the sum (41) can be put under the form $\bar{\pi}^{\prime}=S_{n} \bar{f}_{1}$ with:

$$
S_{n}=\bar{\pi}_{0}+\epsilon q^{-1} b_{12}^{-1} z_{2} \bar{\pi}_{2} .
$$

¿From the definition of $\bar{f}_{1}$, the only way $S_{n} \bar{f}_{1}$ can be symmetrical is when it is equal to zero. Since the kernel of $\bar{f}_{1}$ are the symmetric polynomials, we therefore need to show that $S_{n}$ (42) is symmetrical under the exchange of $z_{1}$ and $z_{2}$. Denoting $b_{j}=b_{1 j}$, and substituting the explicit expressions (37, 39) of $\bar{\pi}_{0}, \bar{\pi}_{2}$, we obtain: 


$$
S_{n}=\bar{\rho}\left(\bar{t}_{\frac{n}{2}} \ldots \bar{t}_{3} \bar{t}_{2} b_{2}+\epsilon q^{-1} \sum_{i=2}^{\frac{n}{2}} \bar{t}_{\frac{n}{2}} \ldots \bar{t}_{i+1} \bar{t}_{i-1}^{-1} \ldots \bar{t}_{2}^{-1} z_{2}\right) b_{2}^{-1}=\bar{\rho} I_{n}
$$

A first application of the affine Hecke relations (27) enables to replace $\bar{t}_{i-1}^{-1} \ldots \bar{t}_{2}^{-1} z_{2}$ with $z_{i} \bar{t}_{i-1} \ldots \bar{t}_{2}$ in (43). A second one gives:

$$
\bar{t}_{i} b_{i}+\epsilon q^{-1} z_{i}=b_{i+1} \bar{t}_{i}
$$

for $i \geq 2$. The repeated application of this identity enables to put the operator $I_{n}$ acting on $\bar{\rho}$ in (43) under the form:

$$
I_{n}=b_{\frac{n}{2}+1} \bar{t}_{\frac{n}{2}} \ldots \bar{t}_{2} b_{2}^{-1}
$$

Using the fact that $\bar{t}_{i}$ commutes with $b_{i j} b_{i+1 j}$, and its action on the product $b_{i+1 j} b_{i i+1}$ :

$$
b_{i+1 j} b_{i i+1} \bar{t}_{i}=b_{j i+n} b_{i i+1}
$$

we obtain a factorized expression of $S_{n}$ (43) exhibiting the symmetry under the exchange of $z_{1}, z_{2}$ :

$$
S_{n}=\bar{\rho}_{n-2}\left(\prod_{k=3}^{\frac{n}{2}+1} b_{1 k} b_{2 k}\right)\left(\prod_{k=\frac{n}{2}+2}^{n} b_{k n+1} b_{k n+2}\right)
$$

where $\bar{\rho}_{n-2}$ is the maximally crossed state of $\mathcal{M}_{n-2}$ expressed in the variables $z_{i}, 3 \leq i \leq n$.

Thus, the relation (38) holds. Let $\mathcal{B}_{n-2}$ be the subalgebra generated by $t_{i}, i \geq 3$. Once we know that the line $\pi_{0}$, of the matrices $\bar{e}_{1}$ and $e_{1}$ are the same, acting with $\mathcal{B}_{n-2}$ on $\bar{\pi}_{0}$ and using the property that $\mathcal{B}_{n-2}$ commutes with $e_{1}$, we can equate the lines $\pi \in \Pi_{0}^{1}$. This establishes that $e_{1}$ projects $\Psi$ onto symmetrical polynomials under the exchange of $z_{1}$ and $z_{2}$ orthogonally to the polynomials divisible by $b_{2}$.

¿From the matrix expression (15), we can extend the definition of $\bar{t}_{1}=t_{1}$ to $\Pi_{0}^{1}$, and thus to the polynomials of $\mathcal{M}_{n}$ not divisible by $b_{2}$. We define $\bar{t}_{i}$ for $i \geq 1$ through $\bar{\sigma} \bar{t}_{i}=\bar{t}_{i-1} \bar{\sigma}$, and this definition agrees with (26) on polynomials divisible by $b_{i i+1} \cdot \bar{t}_{i}$ and $\bar{e}_{i}$ constructed with this procedure are identified with $t_{i}$ and $e_{i}$

To complete the identification we must verify that $\bar{\sigma}(30)$ is equal to $\sigma$ (13). We observe that $\bar{\rho} \bar{\sigma}=\bar{\rho}$, in agreement with the fact that the line $\rho$ of the matrix $\sigma$ has only zeros except a 1 for the diagonal element. Since $y_{1}=\sigma^{-1} \bar{\sigma}$ commutes with $\mathcal{B}_{n-1}$, the algebra generated by $t_{i}$ for $i \geq 2$, and $\mathcal{H}_{n}$ is an irreducible module over $\mathcal{B}_{n-1}$, we deduce that $y_{1}=1$. 


\subsubsection{The cyclic case}

In the cyclic case $p^{-1}=q^{2}$, following the line of [8], it is straightforward to verify that $\Psi$ is the perron-Frobenius eigenvector of a statistical transfer matrix. The Pfaffian state (4) is a symmetrical polynomial obeying the constraint (31), and therefore realizes a trivial representation of the B.M.W algebra.

We can verify this property in the tangle representation. Another way to write the basis elements (9) is by dressing the Temperley-Lieb words with braid generators. The basis is be obtained from the Temperley-Lieb basis [1] by substituting the $e_{i}$ for $i<d_{2 p+1}$ sitting at the end of the sequence $e_{c_{2 p+1} \ldots e_{2 p+1}}$ with $t_{i}^{-1}$ :

$$
\pi=\left(t_{c_{n-1}}^{-1} t_{c_{n-1}+1}^{-1} \ldots e_{d_{n-1}} \ldots e_{n-2} e_{n-1}\right) \ldots\left(t_{c_{2 p+1}}^{-1} t_{c_{2 p+1}+1}^{-1} \ldots e_{d_{2 p+1}} \ldots e_{2 p} e_{2 p+1}\right) \ldots e_{1},
$$

where $c_{2 p+1} \leq d_{2 p+1} \leq 2 p+1$ and $c_{n-1}>c_{n-2}>\ldots>c_{3}>1$. In this presentation, it is clear that when $a=\tau=1$, the generators $e_{i}$ transforms a basis element into another basis elements. Therefore, the matrix elements of each columns of the matrix $v$ (16) are all equal to zero except for a single one which is equal to one. Consequently, the sum of dual vectors $\sum_{\pi} \bar{\pi}$ form a trivial representation and is proportional to (44).

\section{A Explicit construction of $\mathcal{M}_{4}$}

Let us construct $\mathcal{M}_{4}$ the dual of $\mathcal{H}_{4}$.

We search for an invariant vector $\Psi$ of the form:

$$
\Psi=\bar{\pi}_{1}\left(z_{1}, . ., z_{4}\right) e_{1} e_{3}+\bar{\pi}_{2}\left(z_{1}, . ., z_{4}\right) t_{2}^{-1} e_{1} e_{3}+\bar{\pi}_{3}\left(z_{1}, . ., z_{4}\right) e_{2} e_{1} e_{3} .
$$

The dual representation is obtained by acting with the generators on the maximally crossed state $\bar{\pi}_{2} \equiv(0,1,0)$ :

$$
\bar{\pi}_{2}=\left(q z_{1}-q^{-1} z_{2}\right)\left(q z_{2}-q^{-1} z_{3}\right)\left(q z_{3}-q^{-1} z_{4}\right)\left(q z_{4}-p^{2} q^{3} z_{1}\right) .
$$

¿From (26) , we obtain $\bar{\pi}_{3} \equiv(0,0,1)=\bar{\pi}_{2} \bar{t}_{1}^{-1}$ and $\bar{\pi}_{1} \equiv(1,0,0)=\bar{\pi}_{2} \bar{t}_{2}$ :

$$
\begin{aligned}
& \bar{\pi}_{3}=p^{-1}\left(p^{2} z_{3}-z_{4}\right)\left(q^{2} z_{3}-z_{4}\right)\left(p^{2} z_{1}-z_{2}\right)\left(q^{2} z_{1}-z_{2}\right)-p \bar{\pi}_{2}, \\
& \bar{\pi}_{1}=q^{-2} p^{-1}\left(q^{4} z_{1}-z_{4}\right)\left(p^{2} q^{2} z_{1}-z_{4}\right)\left(p^{2} z_{2}-z_{3}\right)\left(q^{2} z_{2}-z_{3}\right)-p^{-1} \bar{\pi}_{2} .
\end{aligned}
$$


¿From these expressions, one obtains the expression of $\bar{t}_{i}$ using (26) and of $\bar{e}_{i}$ by projecting $\bar{\pi}_{j}$ onto a polynomial symmetrical under the exchange of $z_{i}$ and $z_{i+1}$.

The matrices representing the algebra $\mathcal{B}_{4}$ (6) one obtains this way coincide with the tangle representation and are given by:

$$
\begin{array}{ccc}
e_{1}=e_{3}=\left(\begin{array}{ccc}
\tau & a & 1 \\
0 & 0 & 0 \\
0 & 0 & 0
\end{array}\right), e_{2}=e_{4}=\left(\begin{array}{ccc}
0 & 0 & 0 \\
0 & 0 & 0 \\
1 & a^{-1} & \tau
\end{array}\right), \\
t_{1}=t_{3}=\left(\begin{array}{ccc}
a & -a \epsilon & 0 \\
0 & \epsilon & 1 \\
0 & 1 & 0
\end{array}\right)
\end{array}
$$

If we define the operator $\sigma$ acting by cyclically permuting the indices of a tangle:

$$
\sigma=a t_{3}^{-1} t_{2}^{-1} t_{1}^{-1}=\left(\begin{array}{ccc}
0 & \epsilon & 1 \\
0 & 1 & 0 \\
1 & -\epsilon & 0
\end{array}\right)
$$

one can verify that its action on $\Psi$ coincides with that of $\bar{\sigma}(30): \sigma \Psi=\Psi \bar{\sigma}$.

In the case $a=1, \tau=1$, and $\bar{\pi}_{\Omega}=(1,1,1)$ is a trivial representation of the B.M.W. algebra.

\section{B The Brauer Algebra}

The Brauer algebra is the rational limit of the B.M.W. algebra obtained in the limit $z=e^{2 \epsilon u}, q=e^{\epsilon b}, p=e^{\epsilon c}$, with $\epsilon \rightarrow 0$. Then, $\tau=2\left(1+\frac{b}{c}\right)$ and the relations (6) become:

$$
\begin{aligned}
& t_{i} t_{i+1} t_{i}=t_{i+1} t_{i} t_{i+1}, \quad t_{i} t_{j}=t_{j} t_{i}, \text { if }|i-j| \geq 2, \\
& e_{i} e_{i \pm 1} e_{i}=e_{i}, \quad e_{i} e_{j}=e_{j} e_{i}, \text { if }|i-j| \geq 2, \\
& t_{i}^{2}=1, \\
& e_{i} t_{i}=t_{i} e_{i}=e_{i}, \\
& e_{i}^{2}=\tau e_{i}, \\
& e_{i} t_{i \pm 1} e_{i}=e_{i}, \\
& t_{i \pm 1} t_{i} e_{i \pm 1}=e_{i} t_{i \pm 1} t_{i}=e_{i} e_{i \pm 1} .
\end{aligned}
$$

In this limit, the $t_{i}$ are transpositions and therefore, the tangles become link patterns where one ignores crossings.

The structure of the matrices $t_{i}, e_{i}(15)$ is simplified. In the formulas (15), one can set $a=1, \epsilon=0, \tau=2\left(1+\frac{b}{c}\right)$ and all the nonzero matrix elements of $v$ are equal to 1 . 
The property $(\mathrm{P})$ is replaced by:

$\left(\mathrm{P}^{\prime}\right): \bar{\psi}\left(u_{i}=u, u_{j}=u+b, u_{k}=u+2 b\right)=0$, if $i, j, k$, are cyclically ordered.

The cyclic identification becomes: $u_{i+n}=u_{i}+c+2 b$ and it imposes that $\bar{\psi}$ vanishes for $\left(x_{i}, x_{j}, x_{k}\right)=(u, u+b, u+2 b),(u, u+c, u+c+b)$ and $(u, u+b, u+c+b)$ when $1 \leq i<j<k \leq n$.

The operator $Y_{i i+1}(22)$ which permutes the coordinates is given by:

$$
Y_{i i+1}(u)=\frac{(b-u)\left(c-u t_{i}\right)+c u e_{i}}{(b+u)(c+u)} .
$$

$Y$ is a solution of the rational Yang-Baxter equation:

$$
Y_{12}(u) Y_{23}(u+v) Y_{12}(v)=Y_{23}(v) Y_{12}(u+v) Y_{23}(u),
$$

and it is normalized so that $Y_{12}(u) Y_{21}(-u)=1$.

$\bar{e}_{i}$ projects onto the symmetrical polynomials under the exchange of $u_{i}$ and $u_{i+1}$, orthogonally to the polynomials divisible by $b_{i i+1}=u_{i}-u_{i+1}+b$. If we restrict to the components $\bar{\psi} \bar{e}_{i}=0$, the action of the projectors $\bar{t}_{i}-1$ and $\bar{t}_{i}+1$ acquire the expressions:

$$
\begin{aligned}
\bar{\phi}\left(\bar{t}_{i}-1\right) & =-b_{i i+1}^{-1} \frac{u_{i+1}-u_{i}+c}{u_{i+1}-u_{i}}\left(1+k_{i i+1}\right) b_{i i+1} \\
\left(\bar{t}_{i}+1\right) & =b_{i i+1}^{-1}\left(1-k_{i i+1}\right) \frac{u_{i}-u_{i+1}+c}{u_{i}-u_{i+1}} b_{i i+1} .
\end{aligned}
$$

Equivalently, the $t_{i}$ can be commuted through the polynomial using the degenerate affine relations:

$$
\begin{aligned}
& u_{i} \bar{t}_{j}=\bar{t}_{j} u_{i} \text { if } i \neq j, j+1 \\
& \bar{t}_{i} u_{i}-u_{i+1} \bar{t}_{i}=c
\end{aligned}
$$

and their action on the identity is: $b_{i i+1} \bar{t}_{i}=-b_{i i+1}$.

The polynomials are no longer homogeneous and the maximally crossed state is given by:

$$
\bar{\psi}=\prod_{i} \prod_{0<k<\frac{n}{2}} b_{i i+k}
$$


with the cyclic identifications understood. The highest degree monomial can easily be obtained in the limit $b=c=0$ and is equal to:

$$
(-)^{\epsilon} \frac{\prod_{i<j}\left(u_{i}-u_{j}\right)}{\prod_{i \sim j}\left(u_{i}-u_{j}\right)},
$$

Where $i \sim j$ means that $i$ and $j$ are linked, and $(-)^{\epsilon}$ is the parity the number of transpositions $t_{i}$ in the reduced expression of the word.

The polynomials can be decomposed as:

$$
\begin{aligned}
\mathcal{M}_{n} & =\mathcal{M}_{n}^{0} \oplus \mathcal{M}_{n}^{1} \oplus \mathcal{M}_{n}^{2} \\
& =S_{0}+b_{12}\left(S_{1}+\left(u_{1}-u_{2}+c\right) S_{2}\right),
\end{aligned}
$$

with $S_{0}, S_{1}, S_{2}$ symmetrical under the exchange of $u_{1}, u_{2}$. Note that $\mathcal{M}_{n}^{0,2}$ have a highest degree monomial even under the exchange of $u_{1}, u_{2}$, while $\mathcal{M}_{n}^{1}$ have an odd highest degree monomial.

We obtain an explicit expression of $\bar{e}_{1}$ as an operator acting on the components $\Pi_{0}^{1} \Psi=$ $\Psi_{0}$ having the two indices 1,2 connected by a link (split). We know that the action of $\bar{e}_{1}$ on $\Psi_{1,2}$ is equal to zero since these components are divisible by $b_{12}$. We also use the property that $\left(e_{1}+\left(\tau-e_{1}\right) \frac{1}{2}\left(1+t_{1}\right)\right) \Psi_{0}=\tau \Psi_{0}$ which simply results from the fact that the highest degree polynomial of $\Psi_{0}$ is even under the exchange of $u_{1}, u_{2}$ as can be seen explicitly from (61). Thus $\Psi_{0} \subset \mathcal{M}_{n}^{0} \oplus \mathcal{M}_{n}^{2}$.

Let us consider the two complementary projectors defined as:

$$
\begin{aligned}
\bar{e}_{1} & =\frac{1}{c} b_{i i+1} \frac{\left(u_{2}-u_{1}+c\right)}{u_{2}-u_{1}}\left(1+k_{12}\right), \\
\tau-\bar{e}_{1} & =\frac{1}{c}\left(1-k_{12}\right) \frac{\left(u_{1}-u_{2}+c\right)}{u_{1}-u_{2}} b_{i i+1} .
\end{aligned}
$$

The normalization is fixed so that $e_{1}^{2}=\tau e_{1}$. When restricted to $\mathcal{M}_{n}^{0} \oplus \mathcal{M}_{n}^{2}, \bar{e}_{1}$ projects onto $\mathcal{M}_{n}^{0}$, the symmetrical polynomials under the exchange of $u_{1}, u_{2}$, orthogonally to $\mathcal{M}_{n}^{2}$. Thus the action of $\bar{e}_{1}$ on $\Psi_{0}$ projects out the $\mathcal{M}_{n}^{2}$ component and keeps the component of $\Psi_{0} \in \mathcal{M}_{n}^{0}$.

We want to identify $\bar{e}_{1}$ with $e_{1}$ using (23). We first verify this identification on a specific line of the matrix $e_{1}$. For this, consider the following tangle $\pi_{0} \in \Pi_{0}^{1}$ :

$$
\pi_{0}=\left(\begin{array}{cccc}
1 & 3 & \ldots & \frac{n}{2}+1 \\
2 & \frac{n}{2}+2 & \ldots & n
\end{array}\right)=t_{2} t_{3} \ldots t_{\frac{n}{2}} \rho
$$




$$
=e_{1} t_{2} \ldots t_{i-1} t_{i+1} \ldots t_{\frac{n}{2}} \rho=e_{1} t_{1} t_{2} \ldots t_{i-1} t_{i+1} \ldots t_{\frac{n}{2}} \rho .
$$

We also have $e_{1} \pi_{0}=\tau \pi_{0}$, and this exhausts all the matrix elements $e_{\pi_{0}}$ of the line $\pi_{0}$ of $e_{1}$. By duality, we must have $\bar{\pi}_{0} \bar{e}_{1}=\sum_{\pi} e_{\pi_{0} \pi} \bar{\pi}$ :

$$
\bar{\rho} \bar{t}_{\frac{n}{2}} \ldots \bar{t}_{3} \bar{t}_{2}\left(\bar{\tau}-\bar{e}_{1}\right)+\sum_{i=2}^{\frac{n}{2}} \bar{\rho} \bar{t}_{\frac{n}{2}} \ldots \bar{t}_{i+1} \bar{t}_{i-1} \ldots \bar{t}_{2}\left(1+\bar{t}_{1}\right)=0 .
$$

Denoting $b_{j}=b_{1 j}$, and substituting the expressions (5863) of $\bar{t}_{1}+1$ and $\tau-\bar{e}_{1}$, the above equality is equivalent to require that:

$$
S_{n}=\bar{\rho}\left(\bar{t}_{\frac{n}{2}} \ldots \bar{t}_{3} \bar{t}_{2}+\sum_{i=2}^{\frac{n}{2}} \bar{t}_{\frac{n}{2}} \ldots \bar{t}_{i+1} \bar{t}_{i-1} \ldots \bar{t}_{2} c b_{2}^{-1}\right)=\bar{\rho} I_{n}
$$

is symmetrical under the exchange of $u_{1}, u_{2}$.

¿From the degenerate affine Hecke relations (59), we have:

$$
\bar{t}_{i} b_{i}+c=b_{i+1} \bar{t}_{i}
$$

This identity enables us to put the operator $I_{n}$ acting on $\bar{\rho}$ in (66) under the form:

$$
I_{n}=b_{\frac{n}{2}+1} \bar{t}_{\frac{n}{2}} \ldots \bar{t}_{2} b_{2}^{-1}
$$

Using the fact that $\bar{t}_{i}$ commutes with $b_{i j} b_{i+1 j}$ and its action on the product $b_{i+1 j} b_{i i+1}$ :

$$
b_{i+1 j} b_{i i+1} \bar{t}_{i}=b_{j i+n} b_{i i+1}
$$

we obtain a factorized expression of $S_{n}(66)$ exhibiting the symmetry under the exchange of $u_{1}, u_{2}$ :

$$
S_{n}=\bar{\rho}_{n-2}\left(\prod_{k=3}^{\frac{n}{2}+1} b_{1 k} b_{2 k}\right)\left(\prod_{k=\frac{n}{2}+2}^{n} b_{k n+1} b_{k n+2}\right)
$$

where $\bar{\rho}_{n-2}$ is the maximally crossed state of $\mathcal{M}_{n-2}$ expressed in the variables $u_{i}, 3 \leq i \leq$ $n$. Thus, the relation (65) dual to (64) holds. 


\section{Temperley Lieb and $k=2$ cancellation conditions}

This appendix is an addendum to [1], where it was observed that in the $k=2$ case, the Hecke generators $e_{i}$ did obey the more constrained Temperley and Lieb algebra relations. We show that this is a simple consequence of the minimal degree hypotheses in the $k=2$ case.

We consider the space $\mathcal{M}_{n}$ of polynomials obeying the condition (P)(31) and of the minimal possible degree. We assume that the generators $\bar{e}_{1}$, projecting $\bar{\psi} \in \mathcal{M}_{n}$ onto a symmetrical polynomial under the exchange of $z_{1}$ and $z_{2}$, obey the Hecke relations:

$$
\bar{e}_{1} \bar{e}_{2} \bar{e}_{1}-\bar{e}_{1}=\bar{e}_{2} \bar{e}_{1} \bar{e}_{2}-\bar{e}_{2}
$$

We denote by $U$ the above projector and we show here that the Temperley and Lieb condition: $U=0$, is realized when $k=2$.

$U$ projects $\bar{\psi}$ onto a polynomial symmetric under the permutations of $z_{1}, z_{2}, z_{3}$ obeying (P). This implies that $\bar{\psi} U$ vanishes if $z_{1}=z, z_{2}=q^{2} z, z_{3}=q^{-2} z$, and therefore, $E^{\prime}(\bar{\psi} U)$ (34) is divisible by $z_{3}-q^{-2} z$. Since $E^{\prime}(\bar{\psi} U)$ does not depend on $z$, it is equal to zero. The restriction of $E^{\prime}$ to $\mathcal{M}_{n}^{0}$ being injective, this implies that $\bar{\psi} U=0$.

\section{References}

[1] V.Pasquier, cond-mat/0506075.

[2] J.Birman and H.Wenzl, Trans. Amer. Math. Soc. 313 249-273 (1989).

[3] J. Murakami, Osaka J. Math. 24 745-758 (1987).

[4] N.Read and E.Rezayi, Phys.Rev.B 23, 16864 (1996).

[5] N.Read and E.Rezayi, Phys.Rev.B 59, 8084 (1999).

[6] B. Feigin, M. Jimbo, T. Miwa and E. Mukhin math.QA/0112127.

[7] B. Feigin, M. Jimbo, T. Miwa and E. Mukhin math.QA/0209042.

[8] P.Di Francesco and P.Zinn-Justin, math-ph/0412031.

[9] A.Knutson and P.Zinn-Justin, math.AG/0503224. 
[10] J.De Gier and B.Nienhuis, math.AG/0410392.

[11] G.Moore and N.Read, Nuclear Phys. B360, 362 (1991).

[12] L.H. Kauffman, Trans. Amer. Math. Soc. 318 417-471 (1990).

[13] F.M. Goodman and H. Hauschild, Affine Birman-Wenzl-Murakami algebras and Tangles in the solid torus, [QA/04111155].

[14] V.Jones Commun.Math.Phys. 125, 459 (1989).

[15] Y.Cheng, M.L.Ge and K.Xue Commun.Math.Phys. 136, 195 (1991). 